\title{
Rhetorical Characteristics of English for Science and Technology
}

\author{
Ping Wei \\ English Department, The Engineering and Technical College of Chengdu University of Technology, China
}

Keywords: Rhetorical, English for Science and Technology (EST).

\begin{abstract}
English for Science and Technology (EST) is noted for the requirement of brevity, logic, formality, accuracy and precision. However, it also contains a large proportion of rhetoric devices. This paper is going to explore rhetoric characteristics of English for Science and Technology (EST) from the perspective of syntactic devices, lexical devices, phonetic devices and figures of speech. It is hoped that the research can promote the readers' understanding of EST and be of help for the efficiency of the exchange of the information and spreading of scientific facts and laws.
\end{abstract}

\section{Introduction}

Unlike other literary forms like poetry, novels, speech etc, English for Science and Technology (EST) aims to state facts and laws. Readers often jump to the conclusion that EST must be dull and difficult. It is commonly believed that the requirement of logic, brevity, formality, accuracy and precision is in the foreground of the characteristics of EST and there is little room for rhetorical devices in EST. However, in order to facilitate the spreading of facts and laws and promote the exchange of information, many authors when writing science and technology articles tend to adopt various rhetoric devices. Based on Feng Cuihua's (2008) study of rhetorical devices, this paper is going to analyze the rhetoric devices in EST.

\section{Approach to the analysis of rhetorical devices}

According to Feng's book English Rhetorical Options (2008), the rhetoric devices can be analyzed and studied from the perspective of syntactic devices, lexical devices, phonetic devices and figures of speech.

Syntactic devices are devoted to the variation of sentence structures for rhetorical effects, such as the usage of Long and short sentence, syntactic scheme of balance, Syntactic scheme of inversion, syntactic scheme of Omission, Syntactic scheme of Addition, syntactic scheme of repetition and syntactic scheme with "it".

Lexical devices mainly deal with lexical options and lexical repetition. The former is the choice of proper words and the latter is the repetition of words.

Phonetic devices concern the rhyme of the language, which is exploited through the sounds of English words.

Figures of speech refer to ways that makes language figurative, which include most familiar devices like simile, metaphor, personification, hyperbole, pun and so on.

Even though her study is mainly based on the exploration of poetry and other literary works, this approach to the analysis of rhetorical devices can also be of reference when we analyze EST.

\section{Rhetoric devices explored in English for Science and Technology(EST)}

This part of paper is devoted to the analysis of rhetoric devices in EST, which is divided in four sections: syntactic devices, lexical devices, phonetic devices and figures of speech. 
Syntactic devices. Preference to Longer sentences. We can explore the syntactic device by exploiting the variation in sentence length. Considering the length of the sentence we can easily identify that EST contains a large proportion of long sentences. Let see more examples:

The authorities in various countries, especially America, have done well to proceed with extreme caution, emphasizing that there should be little cause for panic, not least because most of the cases have turned out to be false alarms or hoaxes.

Other researchers are bypassing the difficulties of laser drilling and concentrating on soft-tissue applications , where lasers offer some significant benefits.

In the example above, the first sentence has 40 words and the second sentence has 20 words. "According to the statistics on 107 million-word-corpus reported by Shanghai Jiaotong University, 21.4 words is the average length of EST sentences, among which those of more than 40 account for $6.3 \%$, while those of less than 7 (including the seven words) only 8.77\%" (Li Xiuhua,2015.).

It can be assumed from the statistics that EST has a tendency to use longer sentences. Unlike the effect of long sentences in literary works, the EST sentences are long due to the fact that the facts and laws must be explained clearly with no room for misinterpretation.

Preference to Passive Voice. We can also explore the syntactic devices by studying the passive voice of EST. In a literary article, active voice are often employed to identify and emphasis the doer the action. Usually, people avoid using too many passives to distract the attention from doer and make the passage monotonous. However, EST contains a large proportion of passive voice due to the fact that sometimes EST needs to focus on the event itself than on the agent and the fact that EST needs to make statements which sound impersonal. Let consider the following examples:

a). It should be mentioned that KCP-F is one of the first FRET FLASH technology.

b). Natural rubber is obtained from rubber trees as white, milky liquid known as latex. This is treated with acid and dried, before dispatched to countries all over the world.

Passive voice is appropriate in the above sentences. In example a), it is not necessary to emphasize the doer's identity or the doer's identity is unknown. By omitting the doer's identity, the subjectivity of the description is largely reduced. And in example b), "natural rubber" are properly emphasized.

Usage of Syntactic scheme of balance. Syntactic scheme of balance are adopted to enhance clarity and coherence. The principal schemes of balance in EST are parallelism and antithesis.

In parallel construction of EST, parallels of words and phrases are more frequent than the parallels of clause and sentences. As is illustrated below:

a) It includes all features of VAG 1552: easy carry, reliable quality, easy operation, quick reaction, etc..

b) The straw bales, it turns out, are all packed tightly inside a series of prefabricated rectangular wooden wall frames, which are then lime-rendered, dried and finally slotted together like giant Lego pieces, called Modcell panels.

c) the purpose: to provide remote electronic banking, schooling, shopping, taxpaying, chatting, game playing, videoconferencing, movie ordering, medical diagnosing...the list goes on.

In example a), the phrase easy carry, reliable quality, easy operation, quick reaction is used in a balanced way to make the characteristics of VAG clear. In example b), "lime-rendered, dried and finally slotted" are the paralleled verbs to add force to the action. Example e) is a typical syntactic scheme of balance by using nine infinitives in a parallel way.

As for antithesis, which is commonly used in literary works, it is not easy to find examples in EST as in literary works. However, a famous sentence of EST does employ this kind of balanced structure to achieve emphasis of the comparison:

d) Like charges repel, unlike charges attract. I

In example d), two pairs of contrasting idea are presented. The first pair is "like" and "unlike". The second pair is "repel" and "attract". This scheme of balance is very effective in conveying the contrasting ideas. And the antithetical statement has become so famous that even middle school students are familiar with the expression. 
Lexical devices. When analyzing lexical devices, the paper will mainly approach the lexical options and lexical repetition. Lexical options in EST are demonstrated by choice of formal words instead of informal or colloquial words and the choice of Abbreviations.

EST is marked with choice of formal words in that it is closely related to scientific theories, technological terms and engineering techniques. Some of the words are derived from Latin or Greek which will make EST more formal. Take an example for instance:

a) Instead of euthanasia, we use the phrase deliberate administration of an overdose of medication to an ill patient at his or her request with the primary intent to end his or her life.

In the examples above, the word "euthanasia" is a medical terms which is more formal than "mercy killing".

The choice of abbreviations can be found in the following example:

b) In September, we asked Leslie Moonres, president and CEO of CBS and head of UPN, Jeff, Zucher, president of NBC Entertainment, and Chris Albrecht, chairman of HBO, to imagine a perfect television schedule.

From the example above, we can see that the choice of Abbreviation largely reduce the length of the sentence and make the sentence clear and brief. So in EST, the abbreviation will substitute the full form which appear later in the passage. As is shown in the examples found in Return to the Moon:

c) One of the scientific missions being considered is the Moon Orbiting Observatory which will be placed in polar orbit 100 kilometers above the surface. MORO will carry a stereo camera which will give three-dimensional image e showing the landscape.

The similar cases of abbreviation can be found in the EST, like DNA (deoxyribonucleic acid), CD (compact disc), TV (television), AI (artificial intelligence), WWW( world wide web).

Phonetic devices. Knowledge of some English phonetic devices is helpful to both writers and readers. By adopting some phonetic techniques writers can convey the idea more effectively and readers can read more smoothly. Sometimes we can find phonetic devices are employed in EST, though not as frequently as in other forms of literary works, which makes EST less boring as people had expected. The most common phonetic devices in EST are alliteration and homeoteleuton. The former is the repetition of initial sound and the latter is the repetition of ending sound.

Usage of alliteration can be shown in the following examples taken from New Science English and EST Reading:

a) Rogue Asteroids and Doomsday Comets: The Search for the Million Megaton Menace that Threatens Life on Earth

b) Even early air transportation was handicapped by high mountain ranges and large bodies of water.

c) Complete and complex building parts are made in the factory and assembling has resulted in a more efficient building process.

e) Einstein called this idea the cosmological constant and referred to it as his "biggest blunder".

In the above examples, Million Megaton Menace, handicapped by high, complete and complex, cosmological constant , biggest blunder the same consonant sound is repeated in the initial places of the phrase, making reading fluent and impressive.

Besides the author's deliberation to use the word sharing the similar beginning, a unique lexical phenomenon also contributes to the category of alliteration since many technical terms shares the same prefix, which is shown in the sentence below:

f)Three types which particularly serve domestic needs are polyvinylchloride(PVC), polyethylene and polystyrene and each of them may be found in different forms which meets different needs

"polyvinylchloride (PVC), polyethylene and polystyrene" practically forms an alliteration. But this kind of alliteration is peculiar to EST in that EST has a lot of new words associated with the development of science and technology, which may share the same prefix. For instance, the prefix tele means "from far", and it is used to form modern EST terms like telegram, telephone, telecommunication, etc.

Another phonetic devices in EST is homeoteleuton. EST differs from other styles in that it has lots of nouns and many of those nouns are derived from a verb. Due to the fact that they may have the 
same suffix, those nouns may have the similar ending. As the result, many words in EST practically have the similarity in ending, which falls into the category of "homeoteleuton" . Here are some examples:

g) Commercial and industrial growth stimulates invention and innovation in agriculture.

h) Given comparable accuracy and efficiency, a scoring system with less variability in length would be preferable.

Invention and innovation in example d) and accuracy and efficiency in example e) are cases of hometeleuton, which were caused by nominalization.

Figures of Speech. By figure of speech, Feng(2008) refers to those commonly used tropes in classic rhetoric. "tropes have to do with the way words are made to mean other than what would normally imply, and therefore involve deviation from the ordinary and literal meaning of words".(Feng, 168). Common figures of speech found in EST are simile, metaphor, personification, etc.

Simile. Simile is the figure of speech that makes comparison between two different things which have at least one feature resembling each other. The most noticeable feature of simile is the marker

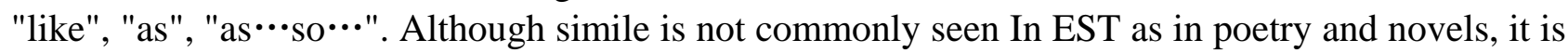
sometimes adopted to make the image sharper and more impressive. For instance, in the sentence "Those tectonic plates are constantly jostling each other, like rafts crowded into a small pond" , the usage of simile like rafts crowded into a small pond vividly describe the way the quakes are born.

Here are more examples:

a) this types of washing machine uses a pulsator to force the water to move like a whirlpool inside the drum.

b) A closer look reveals that the borders of the set do not form crisp lines but seem to shimmer like flame.

In example a), by saying "like a whirlpool", we adopt simile to compare the two unlike elements "pulsator" and "whirlpool" which have the similar feature: the way they force the water to move. The movement of up and down was easily described and understood. And in example b) the borders of the set was compared to flame to emphasize the resemblance that both of them shimmer.

Metaphor. Metaphor is a figure of speech that makes comparison between two different things. Unlike simile, the comparison is implied rather than introduced by words like "like". Metaphor is a common pin EST. For instance, the word "mouse" in the sentence "Use the mouse to drag the icon to a new position" means a small device that moved by hand across the surface to control the cursor of a computer, which is a metaphor. When people see the word mouse, they will visualized the image of that small animal moving swiftly in the field or room and understand the feature of that small computer device without much explanation.

More examples are listed below:

a) Think of how the tall buildings in New York could perform in a symphony of colours as the glass in them is made to change colours instantly.

Here the word symphony associates the fast change of the rich color with a long complicated piece of music for orchestra.

b) standard and protocols set the speed limit on the highway, assign rights of way, deal with mistakes. etc.

Example b) deals with computer and information technology. Highway here makes readers associates the resemblance of the rapidity of information exchange with high speed of traffic on superhighway.

Metaphor can be found in the usage of verbs and verb phrases as well, as is showed in the sentence below:

c) American buildings will pancake.

d) Dark energy adds fuel to the fire of believers in multiple universes, or the idea that our own existence is just one of countless worlds in which the constants and conditions are different.

e) These emails are carefully designed to prey upon those who may be victims of the current recession and tough economic times. 
In example c) the verb pancake is used to compare the image of earthquake-shattered buildings to a thin flat cake, which is vivid and easy to understand. In example d) and e), the verb "prey" and verb phrase "add fuel to the fire" are also used figuratively.

Metonymy. Metonymy is a figure of speech that "has to do with the substitution of the name of one thing for that of another. This substituted name may be an attribute of that other thing or be closely associated with it." (Feng Cuihua, 195). This figure of speech can be illustrated in the example Repeated magnification of the borders plunged one into a bottomless phantasmagoria of Baroque imagery. In this sentence, Baroque is the substitution of Baroque's style.

Allusion. Allusion is a figure of speech which is associated with famous people, things or events in classic books or in history. By using allusion, author assumes that the readers might be quite familiar with the names or events and appreciate their significance. Here is an examples:

a) Earth and its two neighboring planets, Mars and Venus, were formed at the same time, about 4.6 billion years ago, from the same ingredients, including water, carbon dioxide and nitrogen. But only Earth develop life. Mars is too cold. Venus is too hot, but Earth is just right, like Goldilocks' porridge .

b) Hercules wire rope.

In example a) Goldilocks' porridge reminds us the story that a little girl called Goldilocks who went inside the house of three bears' and ate three bowls of porridge. In EST, sometimes you can discover familiar allusions form English tales and legends. In Example b) the features of wire rope is associated with the legend story of Hercules.

Personification. Personification is a figure of speech that endows inanimate objects with human feelings, actions or other characteristics unique to human beings. Personification is used in EST to make it less difficult to understand. Let see some examples:

a) Pollution lingers at Old Missile Site.

The word "linger" are used to give pollution a human attribute. This is a title of an article exploring events of public security. By using personification, average people might have the interest or patient to move on with the article.

b) Hurricane Isaac Pounds Gulf Coast

In the above title, the word "pound" was used to imitate the human action. And the National Weather Service of the United States gave the hurricane a boy's name, making it more personified.

\section{Conclusion}

Science and technology are undergoing rapid progress and have exerted profound influence on EST. EST has developed its own characteristics which distinguished it from everyday English and English used in literary works. This paper explores characteristics of EST from the rhetorical perspectives, which elaborates the characteristics of syntactic devices, lexical devices, phonetic devices and figures of speech in EST. By doing so, it is hoped that readers or translators of EST can have a better understanding of EST and promote the exchange of scientific and technical information.

\section{References}

[1] Changzheng Zou. New Science English. Beijing: Higher Education Press, 2007

[2] Cuihua Feng. English Rhetorical Options. Beijing: Foreign Language Teaching And Research Press, 2008.

[3] Hongmei Han. Selected Reading on Disasters. Beijing: Tsinghua University Press, 2013.

[4] Qinggui. Chen. EST Reading. Beijing: Higher Education Press, 2011

[5] Xiuhua . Li \& Li Li. Characteristics of English for Science and Technology, 2015 International Conference on Humanities and Social Science Research, 2015. 